\title{
Linked Data for Fighting Global Hunger: Experiences in setting standards for Agricultural Information Management
}

\author{
Thomas Baker and Johannes Keizer
}

\begin{abstract}
FAO, the Food and Agriculture Organization of the UN, has the global goal to defeat hunger and eliminate poverty. One of its core functions is the generation, dissemination and application of information and knowledge. Since 2000, the Agricultural Information Management Standards (AIMS) activity in FAO's Knowledge Exchange and Capacity Building Division has promoted the use of Semantic Web standards to improve information sharing within a global network of research institutes and related partner organizations. The strategy emphasizes the use of simple descriptive metadata, thesauri, and ontologies for integrating access to information from a wide range of sources for both scientific and non-expert audiences. An early adopter of Semantic Web technology, the AIMS strategy is evolving to help information providers in nineteen language areas use modern Linked Data methods to improve the quality of life in developing rural areas, home to seventy percent of the world's poor and hungry people.
\end{abstract}

\section{Agricultural information and Semantic Web}

The Food and Agriculture Organization (FAO), headquartered in Rome, is a specialized United Nations agency leading international efforts to defeat hunger. FAO serves as a neutral forum for discussing policy and agreements aimed at ensuring good nutrition through improving agriculture, forestry, and fishery practices, with special attention to developing rural areas, home to seventy percent of the world's poor and hungry people.

Thomas Baker

Washington DC, USA

Johannes Keizer

FAO, Viale delle Terme di Caracalla, 00153 Rome, Italy 
One of the primary tools in FAO's fight against hunger and poverty is Knowledge, and FAO has defined itself as a Knowledge Organization. FAO collects, analyzes, interprets, and disseminates up-to-date information on nutrition, food, and agriculture in a variety of genres and formats - from statistics and databases to bibliographies and workshop proceedings - for an audience of decision makers, technical specialists, agricultural "extension workers," and end users (farmers) in 190 member countries and territories around the world.

With six technical departments in Rome, each with a distinctive disciplinary culture, and field offices in many countries, FAO shares the knowledge management challenges common to any complex, global organization, with the additional challenge of targeting an audience in areas that are poor in resources and IT expertise and that require the use of many local languages in the service of end users that may be illiterate.

This chapter assesses the experience of the Agricultural Information Management Standards (AIMS) activity in FAO's Knowledge Exchange and Capacity Building Division over the past decade in promoting the use of Semantic Web standards to improve the dissemination and use of information on nutrition and technical innovation in agriculture. It is based on meetings and interviews held in 2009-2010 for an "autoevaluation" undertaken to critically as\$ess the achievements, impact, and strategic direction of this activity at the start of a new programme cycle.

The story begins in the early 2000 s, when a series of workshops with experts and international partners encouraged FAO to work with Member Countries to become "a key enabler and catalyst to establish a new model of agricultural information management in the 21 st century" based on decentralized information management and using "Web-enabled" standards for interoperable data exchange. The guiding theme was provided by Tim Berners-Lee's seminal keynote at XML2000 1 outlining his vision of a Semantic Web based on "ontologies." Under the banner "Agricultural Ontology Server" (AOS), and supported by the Agricultural Information Management Standards (AIMS) community Website 2 , a team in the Knowledge Exchange Facilitation Branch (KCEW) at FAO developed a program with three main components:

-The use of simple descriptive metadata for integrating access to agricultural information in both developed and developing countries and, to a lesser extent, in FAO's own technical departments.

-The development and maintenance of thesauri and ontologies - especially FAO's flagship vocabulary of agricultural terminology, AGROVOC 3 — as descriptors for structuring access to agricultural information and as "building blocks" for application-specific ontologies.

- Networking, capacity development, and outreach aimed at promoting the uptake and use of these standards by FAO information providers and partner organizations.

\footnotetext{
${ }^{1}$ http://www.w3.org/2000/Talks/1206-xml2k-tb/

2 http://aims.fao.org/

${ }^{3}$ http:/aims.fao.org/website/AGROVOC-Concept-Server/sub
} 
As an early adopter of Semantic Web technology, the AIMS team has been years ahead of the curve in porting its legacy information management standards from the print world into Web formats and is well-positioned to benefit from current technological trends. In some areas, however, the team is paying a price for having been a bit too far ahead of the curve. This chapter summarizes the work done, lessons learned, and outlines some course corrections decided as a result of the autoevaluation:

- The concept of application profile it used has allowed the AIMS team (and others) to merge information from diverse sources into central databases but now needs to be loosened to accommodate input that is either simpler (where resources are scarce) or more complex (where requirements are more comprehensive) - something which more flexible technological approaches now support.

-The metamodel custom-designed in-house for upgrading AGROVOC and other AIMS thesauri into Web-enabled ontologies, while novel and innovative in 2004, has been superseded by an international standard that serves the same function but with the promise of tool support and compatibility with a rapidly growing number of other Web-enabled vocabularies.

\section{Promoting trusted URIs for use in Linked Data}

The Semantic Web vision outlined in 2000 achieved its breakthrough when Tim Berners-Lee radically redefined the message in 2006 around the notion of Linked Data4. The term Linked Data refers to a style of publishing structured data on the Web in which all elements of an ontology (properties, classes, and value vocabularies), as well as things described by the ontology (publications, events, people), are identified by Uniform Resource Identifiers (URIs), allowing data to be extensively cross-referenced ("linked") with other data sources.

The vision of Linked Data is succeeding where Semantic Web did not because it conveys a simple message that can be understood in very concrete terms. People can see that it has to do with how things relate to each other and about making such links resolvable on the Web for practical purposes such as structured browsing and data integration.

In Linked Data terms, an ontology is a conceptual structure represented as data. Services can be built over that data. Using HTTP URIs and resolving those URIs to useful information that people can look up replicates the function of a dictionary. By promoting use of the URIs of AIMS standards for tagging (annotating) Web content worldwide, AIMS can empower resource providers to bypass centralized aggregators and search engines, which seek to position themselves as gatekeepers, and connect their resources directly to a growing Linked Data cloud. URIs provide language-neutral hooks for labeling shared concepts in any of the languages used

\footnotetext{
${ }^{4}$ http://www.w3.org/DesignIssues/LinkedData.html
} 
in FAO member countries, enabling coherent access to information across language areas.

As the technological approach which AIMS helped pioneer now matures, AIMS will be able to benefit from generic software tools developed in the commercial world and open-source communities. With mainstream search engines and applications adopting the Linked Data approach, AIMS can transition from the role of technological innovator to that of developing capacity to help information providers in member countries benefit from the Web revolution.

The sections which follow review technical achievements, user feedback, and planned course corrections with respect to:

-Metadata based on application profiles that use open, Semantic Web vocabularies to describe documents and other objects of interest, such as events, people, and learning materials.

- Thesauri such as AGROVOC, upgraded for publication and use in a networked environment, and their alignment with specialized vocabularies in domains such as fisheries.

- Collaboration among partner organizations in the creation, maintenance, and deployment of standards for sharing knowledge related to food and agriculture, notably in the context of an umbrella initiative, Coherence in Information for Agricultural Research for Development (CIARD).5

All of the standards and projects discussed below are documented or linked on the AIMS Website. 6

\section{Integrating access using Dublin Core metadata}

Work on the standards that now fall under the banner of AIMS began under an Agricultural Metadata Standards Initiative (AgStandards) in 2000. Inspired in part by the Dublin Core Metadata Initiative, then five years old, the AgStandards Initiative took the fifteen elements of the Dublin Core Metadata Element Set (DCMES) - basic elements such as Title, Subject, and Date - as a starting point and defined itself as an umbrella under which additional elements could be created. A new namespace for describing document-like resources relevant to agriculture, Agricultural Metadata Element Set (AgMES), was published in 2005 as the first output of the initiative.

The flagship implementation of AgMES is the International Information System for the Agricultural Sciences and Technology (AGRIS), FAO's database of bibliographic references to literature produced by agricultural research centers around the world. From its beginnings in 1969 - the name "AGRIS" dates from 1975 through the late 1990s, AGRIS was maintained by FAO as a centralized database with its own unique database structure, exchange formats, and software.

\footnotetext{
${ }^{5} \mathrm{http}: / \mathrm{www}$. ciard.net/

${ }^{6}$ http://aims.fao.org/
} 
With the rise of the World Wide Web and its new paradigm of distributed information management, the AGRIS database was by 2000 looking old-fashioned and unsustainably centralized. Between 2000 and 2003, a series of workshops with experts and international partners encouraged FAO to diversify institutional participation in AGRIS through capacity development, which aimed at empowering local and regional AGRIS centers to improve information management in their own institutions. The workshops endorsed the role of FAO in supporting common standards and protocols for achieving this goal.

The renewed AGRIS effort focused on the use of a simple application profile based on Dublin Core - the AGRIS Application Profile - as the basis for conversions from a wide range of local database formats into a common XML format (Document Type Definition, or DTD). To facilitate the adoption of the AGRIS profile by AIMS partners such as the Global Forestry Information Service and the research centers of the Consultative Group on International Agricultural Research, the AGRIS team defined mappings from legacy data formats and developed simple data input tools ("WebAGRIS" and "MetaMaker").

The AGRIS Application Profile, which was originally designed published with an RDF variant, was intended from the start as a means for gathering data from partners that could be expressed in triples. The problem was that most AGRIS partners were and continue to be unprepared to generate RDF data on their own. The AGRIS DTD served as an aid for generating a repository of data that could straightforwardly be converted into RDF.

By 2005, the AGRIS team had converted the entire repository of three million records from its legacy library-catalog-based "AGRIN3" format into XML records based on the AGRIS profile. Over the years, data has accumulated in AGRIS from over two hundred institutes, and of today's one hundred AGRIS providers, roughly sixty remain "very active." Some AGRIS data is delivered by motorbikes over dirt roads on thumb drives. Institutions have been encouraged to configure their databases to generate conformant XML data for harvesting and transformation by the central AGRIS team. The introduction of the AGRIS AP as a common exchange format dramatically reduced the need for editing and cleaning incoming data, which before 2000 had been done by a team of more than ten people at the AGRIS processing unit in Vienna.

The AIMS team followed up its publication of the AGRIS profile by developing or promoting profiles for other types of information - e.g., for News (using the standard RSS news format) and Events (a simple profile with starting and ending dates, location, type, and organizer). These were used for an alert service, AgriFeeds7, which was launched in 2007. The team also created a profile for brief descriptions of organizations which, when published on their own Websites in XML, can be referenced in metadata or harvested for automatic compilation into lists.

In 2006, work began on a profile for providing structured access to learning resources in a Capacity and Institution Building Portal. This profile uses results from

\footnotetext{
${ }^{7} \mathrm{http} / /$ www.agrifeeds.org/

${ }^{8} \mathrm{ftp} / / \mathrm{ftp}$.fao.org/docrep/fao/010/ai154e/ail54e00.pdf
} 
an ongoing effort by DCMI and the Institute of Electrical and Electronics Engineers (IEEE) to harmonize the simpler approach of Dublin Core metadata with the more comprehensive and complex specification of the IEEE Learning Object Metadata standard on the basis of a Linked-Data-compatible representation.

\section{Feedback from application profile users}

The renewal of the legacy AGRIS database as a Web repository is generally seen as a big success, and the AgriFeeds service is widely used. The repository has exposed local research results to a global audience. The AGRIS center in South Korea, for example, has been delighted at the surge in requests for its publications, especially since AGRIS has been picked up by Google.

The AGRIS Application Profile 1.1 of July 20059 , however, prints out at eightyone pages, and as various users attest, the profile is widely perceived as "heavy" and "cumbersome" to implement, requiring a higher level of control than users in low-budget situations can afford:

We do not use the AgMES application profile. Not that we reject it, but we see that such applications are too heavy-duty for people in developing countries. They do not have the staff to do detailed things, and we do not want to push them to adopt anything. At our home office we have even less capacity for adding metadata or mapping.

An AIMS partner confirms that even the task of mapping from existing formats presents a significant barrier:

Today we have over 170 information provider parthers from around world, but only half have created RSS feeds links to us - and only because we could show that it did not take much working time. We have had even less success in getting partners to create AGRIS data from their native records - it is a bigger job for them to understand the records and make the mapping.

A minority of users see the problem less as one of excessive complexity than of excessive simplicity and lack of flexibility. Work on an application profile for describing projects ran up against the limits of simple, flat (and therefore more easily interoperable) descriptions with the need to provide contact information for project coordinators and recipient institutions - information that requires descriptions about additional entities, such as people and organizations, to be embedded in records about publications.

However, a larger number of users would prefer to see AGRIS lower the bar by promoting simpler, lighter alternatives, perhaps even using just a handful of Dublin Core elements:

In order to justify the working time, our information providers want to see how this will help them get more users, like offering a simple search tool. Maybe FAO could make the profile simpler and more flexible. Start with something very simple, like RSS, before introducing more comprehensive metadata solutions.

\footnotetext{
${ }^{9}$ http://www.fao.org/docrep/008/ae909e/ae909e00.htm
} 
We would like to submit data to AGRIS. The problem is that the data is very dirty - it is collected from different sources. The funder collects things they no longer fund, and you have to accept everything and get very dirty metadata. We require something a bit lighter than the AGRIS application profile.

AGRIS staff point out in response that "the AGRIS profile is perceived as complicated because people see the fifty or sixty fields but do not realize that only five or six of those fields are mandatory." The AGRIS team does in fact accept data in whatever granularity it is provided. Many descriptions provide just a minimum, with Title, Subject (typically with an AGROVOC value), Date, Availability (location), Language, and often Conference Name. This message, however, has not been widely understood, so future Web guidelines and training sessions will highlight simple examples.

AGRIS staff also note that the role of metadata is shifting in ways which deemphasize the importance of information about the location of a resource. In the Web world resources are, in practice, often moved around or replicated on multiple servers. Google, on the other hand, excels at finding "known entities" - resources for which an exact title, authors, or other publication information is known if not the location. In the new division of labor between search engines and curated collections, bibliographic databases can help users discover that a resource exists, then Google can help them find and retrieve it. One user suggests that, if nothing else, tagging resources by subject would by itself be a big win:

Focus less on application profiles than on using AGROVOC well. If people could pull elements from AGROVOC just to tag their things, it would be fantastic.

Other users caution, however, that even minimal requirements can be hard to meet. They report that "with the AGRIS profile, people are sometimes intimidated by the big words, even if it is just their own data fields that are getting mapped." The underlying problem, according to many, is the lack of basic information management skills:

\footnotetext{
In our experience with RSS and the AGRIS profile, the main problem is not with the specifications themselves. The biggest problem is that organizations which maintain and create information on the Web do not have knowledge or skills to maintain metadata. They have old-fashioned Web sites - hand-made, not dynamically generated. Behind those Web pages, some developers have learned to maintain Web pages, but the structure as a whole is not well prepared. Only a few providers know how to create RSS or AGRIS XML data, upload to the Website, and link to our service.
}

The solution, expressed in many ways by the people interviewed, lies in capacitydeveloping measures for bringing users up to speed with the technology:

\footnotetext{
Ninety percent of our users are in developing countries. The key is capacity building. It is one thing to publish a specification, but to get uptake in twenty institutions, you need to hold face-to-face meetings, identify champions, and train the trainers.
} 


\section{Metadata enrichment and conversion to Linked Data}

The AIMS team is currently exploring ways to leverage AGRIS in the Web environment by publishing the entire repository in the form of RDF "triples" - the fundamental unit of Linked Data. The process involves "metadata enrichment" - the progressive enhancement of descriptions, where possible, with explicit links (URIs). This turns each AGRIS record into an entry point to a web of authors, institutions, and topics - a "hub" for drawing together a global collection of information and, by extension, the community of its authors.

The new role of URIs in weaving the Web changes the role of metadata itself by de-emphasizing its function for finding information, for which people often turn to Google. Rather, metadata functions increasingly as a bundle of links that embed a given resource in a web of relationships, thereby giving that resource a context.

With help from the information management company Talis and a team from the Okkam Project 10 at the University of Trento, the AGRIS team is testing the "triplification" of AGRIS XML records. Talis is testing the conversion of string values for Creator, Publisher, Language, and Type into URIs from authority files for authors, journals, languages, and resource types. The Okkam Project is testing algorithms for disambiguating between authors, given inconsistently entered names, by using contextual information such as affiliation, co-authorship, or country. Subject, arguably the most important field in AGRIS descriptions because it links resources to FAO's areas of interest, is also one of the "cleanest" in the dataset because it was populated largely using tools which copy subject strings directly from AGROVOC online.

Before the conversion of strings into URIs, data must often first be cleaned by normalizing variant strings to the "termspell" (normalized string) of a target vocabulary. The process of cleaning, normalizing, and enriching cannot be fully automated — people need to control the results at every step — and the procedure is intended to be a one-way migration, not something that is carried out repeatedly and on-the-fly. It greatly helps that the XML data files of AGRIS are already partitioned according to year and month of ingest, country, and institution because the quality of records systematically improyed as AGRIS centers acquired better data-entry tools.

Moving forward, the AGRIS team aims at facilitating the use of URIs by increasing tool support. AIMS partners are developing small utilities and plug-ins, for example, to tag content with AGROVOC descriptors ("AgroTagger"), enhance string-based record fields with URIs in DSpace repositories, and identify concepts in texts for annotation with URIs in Drupal content management systems ("AgroDrupal"). As one AGRIS manager explained, the AGRIS profile can be taken as a foundation and, starting with a minimal record, tools can be used to enrich the data, automatically, with information extracted from the content of the resource or inferred from its context.

${ }^{10} \mathrm{http}: / /$ www.okkam.org/ 


\section{Accepting "whatever you can get"}

For many years, the dominant paradigm for the interoperability of digital information has been syntactic conformance with specific data formats encoded as XML DTDs or XML Schemas. AIMS application profiles were based on a set of welldefined data elements semantically compatible with RDF properties and classes. Transforming AGRIS partner data into the AGRIS XML format was a process of mapping local data elements of AGRIS data providers to common target elements. As the concept of Linked Data had not been developed in 2005, and most AGRIS partners lacked and continue to lack the experience for publishing their data directly in an RDF representation syntax, the AGRIS DTD has served as a transitional aid for creating data that is conceptually and semantically (though not syntactically) interoperable with RDF.

The emerging paradigm of Linked Data, in contrast, explicitly avoids requiring that information providers expose identical formats. RDF provides an abstract model for data that can be serialized in one of several interchangeable syntaxes for representing data as generic "statements" (RDF "triples") that can be joined automatically on the basis of shared global identifiers (URIs). The "Open World Assumption" underlying Linked Data avoids assuming that any one source provides complete and exhaustive information about a given resource and anticipates that information sources may only partially overlap. Whereas formats such as DTDs can be "broken" by omitting data, triples constitute a language in which "missing is not broken" [1]. By anticipating the future integration of new sources even if they are not completely aligned, the architecture of Linked Data is more resilient to imperfections and diversity, while the syntax-independent model of triples makes data more "future-proof."

In the new paradigm, interoperability is an unbroken continuum that depends on the "coherence" of merged triples. Coherence is provided best by shared URIs - URIs for identifying the resources described, for naming the properties used to characterize the relationships between resources, for citing the classes used to characterize types of resource, for defining the datatype of string values, and for characterizing values as members of specific controlled vocabularies. Taken together, these URIs serve to "qualify" data by putting its values into the context of known standards. Qualified data can more easily be integrated across multiple sources because URIs provide a firm basis for alignments and mappings.

String values - sequences of alphanumeric characters such as names, dates, and publication abstracts - are inherently less precise as a basis for merging data due to natural variations in spelling or punctuating subject headings and titles, representing names, or formatting dates. To improve their value for Linked Data, it is important that string values be qualified, when possible, with descriptive context. Date strings, for example, can be expressed as RDF datatypes (in Dublin Core terminology, Syntax Encoding Schemes) by providing a URI identifying the ISO or W3C standard that specifies the pattern used for sequences of months, days, and years.

Value vocabularies are most effective for use in Linked Data when their individual terms are identified using URIs, as with AGROVOC. However, a URI identifying 
a Vocabulary Encoding Scheme, or VES (in Dublin Core terminology) can be used to put a string value into the context of a controlled vocabulary. Using a VES URI together with a string is not as precise as using a URI for a specific term, but for controlled vocabularies that have not yet been "Webified," it is better than providing no context at all.

Shifting the emphasis from shared data formats to the coherence of underlying triples will allow the AGRIS team to relax the requirements for data ingest and more flexibly accommodate data from a growing diversity of providers. Providers using RDFa to embed structured descriptions "invisibly" into normal Web pages, for example, will be able to use tools such as Yahoo SearchMonkey to extract the underlying triples for ingesting into AGRIS. This shift redefines the function of the AGRIS DTD, and other such constructs, from that of ensuring interoperability through uniformity of format to that of providing a validatable template that is cleanly convertible into RDF triples. In the context of Linked Data, templates and application profiles of this type will continue to ensure that data are created with enough "qualification" to support more-precise, higher-quality data integration.

\section{AGROVOC and specialized domain ontologies}

AGROVOC, a multilingual thesaurus of agricultural topics, was created by FAO and the Commission of the European Communities in the early 1980s. It consists of "terms" (natural-language phrases) in multiple languages cross-referenced with other broader, narrower, and related terms. The thesaurus standardizes term codes and "termspells" (spelling and punctuation) in order to improve the quality of indexing and search.

From 8,660 descriptors (preferred terms) in 1982, AGROVOC grew to 16,607 descriptors by 2000 and has roughly 32,000 descriptors today. Initially available in English, French, and Spanish, AGROVOC is now available in nineteen languages, with additional translations in the works. Periodic releases of AGROVOC can be freely downloaded in its native relational database format or in alternative formats such as Microsoft Access, and the latest version can be accessed by applications via Web services for looking up terms or expanding queries. AGROVOC terms have been mapped to terms in the Chinese Agricultural Thesaurus, the Schlagwortnormdatei Thesaurus of the German National Library, the US National Agricultural Library Thesaurus, the General Multilingual Environmental Thesaurus of the European Environment Information and Observation Network, and the CAB Thesaurus of the UK-based technical agency CAB International.

In 2001, the (future) AIMS team envisioned an Agricultural Ontology Server as "a reference tool that structures and standardises agricultural terminology in multiple languages," providing modules of terms that can serve as "building blocks" for developing more specific domain ontologies. Starting in 2005, the AIMS team focused on "refining" AGROVOC's standard thesaurus relationships ("Broader Term," 


\section{Agrovoc OWL model today}

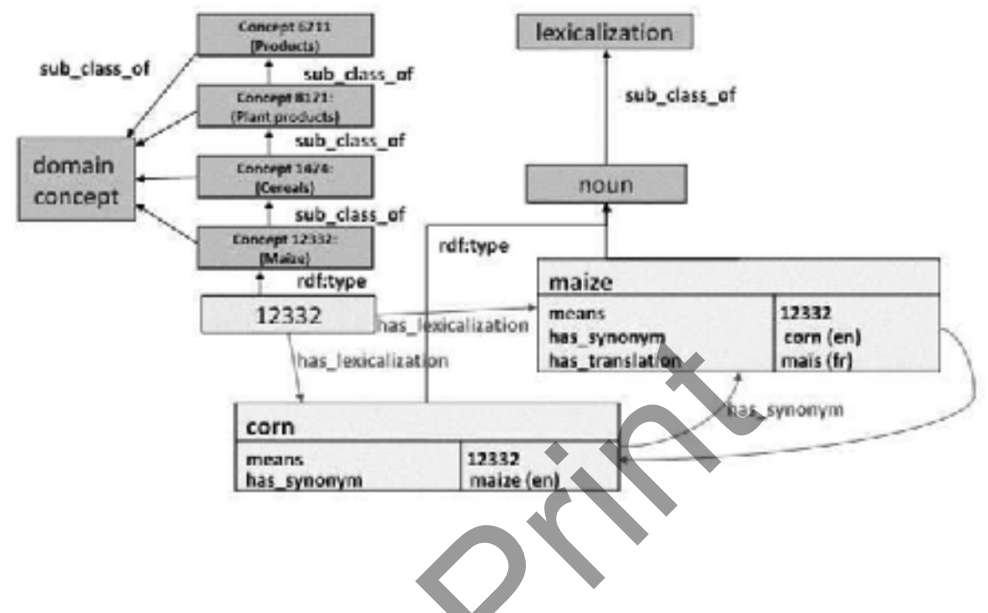

Fig. 1 Metamodel for the AGROVOC Ontology, 2006-2010 (simplified)

"Narrower Term," "Related Term," and "Used For") into semantically more specific relationships such as "hasIngredient" or "growsIn." 11

This refinement of thesaurus relationships was undertaken with the implicit assumption that a more precisely engineered ontology would support more intelligent queries - for example, to determine whether a specific farming method has been used in a dryland area for a given crop and to find any relevant research reports in whatever language they may be available. Most of the refinements have been defined by experts at the International Crops Research Institute for the Semi-Arid Tropics (ICRISAT) in Patancheru, India.

The AGROVOC project team formulated a conceptual model with "the necessary structure to create precise semantics to facilitate the transition from traditional thesauri to ontologies" — in effect a "metamodel" for thesauri. [10] In the final form of the metamodel (see Fig. 1):

-The natural-language Terms of the AGROVOC Thesaurus are re-conceptualized as Lexicalizations (Labels) for underlying Concepts. Lexicalizations include preferred and alternative labels, synonyms, spelling variants, and translations

\footnotetext{
${ }^{11} \mathrm{http} / /$ agrovoc.icrisat.ac.in/agrovoc/relationstree.php
} 
in multiple languages. Descriptors are conceptualized as "preferred" Lexicalizations.

-Concepts are modeled as OWL Classes (i.e., as sets of things). [7]

-Each Concept-Class is associated with one Instance of that Class as a means of relating a Concept to its Lexicalizations. (This was done to meet a perceived need for description-logic-based computability, as declaring one Class to be an Instance of another Class sacrifices conformance with "OWL DL," a constrained, description-logic-conformant sub-set of the more expressive but computationally intractable variant “OWL Full.”)

-Relationships can also be specified between Concepts (such as "isUsedIn" or "causes") or between Lexicalizations (such as "hasAcronym"). In 2006, this was considered a significant and innovative feature of the metamodel.

Converting the metamodel of AGROVOC into a class-based ontology, however, was only part of the AIMS vision. Equally important was the notion of enabling AGROVOC to evolve dynamically, in response to technical innovation, scientific advances, regional specialization, and linguistic evolution. Just as AGRIS member institutions were empowered to submit bibliographic data directly, decreasing dependence on the central team in Rome, there was a strong push to enable expert users in AGROVOC's twenty-some language areas to maintain the ontology directly online. Aside from relieving the central AGROVOC team of the cumbersome and relentless task of processing change requests - a frustrating bottleneck both for the team and for its users - the idea of moving maintenance to the Web addressed what Martin Hepp refers to as the trade-off between "ontology engineering lag versus conceptual dynamics" [4] — the insight that knowledge itself is continually evolving, that the process of ontology development is necessarily iterative and dynamic, and that for semantic applications, the most important concepts are frequently also the newest.

In 2005, requirements were developed for a Web-based platform - the AGROVOC Concept Server Workbench - to allow experts in many countries to add or translate concepts in their specific areas of interest. The Workbench was conceived as a distributed, Web-based maintenance environment that would enable participants in multiple countries to edit parts of the central AGROVOC ontology simultaneously — adding term translations, adding or refining relationships between terms, or performing batch modifications on the basis of pattern matching. The Workbench was also seen as a platform for plug-in tools that could proactively populate AGROVOC with new concepts extracted by corpus analysis from breaking news stories ("ontology learning"). The move to a distributed architecture was seen as a way to loosen the dependence of AGROVOC on terms entered canonically in English, then "translated" into other languages, towards an environment in which users could create new locally-specific terms in any language.

The system was intended to support levels of authorization ranging from Guest Users through Term Editors, Ontology Editors, Validators, and Publishers, to System Administrators. It was designed to support the extraction and export of sub-sets of concepts for personal use and the upload of entire ontologies for sharing with others. It was conceived of as a generic tool in principle adaptable to other domains, 
such as health care and medicine. Part of the vision was eventually to provide addon services such as automatic or semi-automatic translation, ontological reasoning, guided search, and concept disambiguation.

In 2006, having formulated Workbench requirements and finalized the OWLclass-based ontology model, the AIMS team, finding no software capable of fully implementing this vision off-the-shelf, undertook the development of a customized interface to a backend ontology database, Protege ${ }_{12}$. This software development project has been led since 2006 by Kasetsart University in Thailand with input from implementation testers in Rome and Patancheru. An alpha version of the Workbench was released in June 2008, and development has accelerated in 2010 with the involvement of a development team at MIMOS Berhad in Malaysia. AGROVOC has in the meantime been maintained in the original thesaurus database, with snapshots periodically exported to the Workbench for testing. After a final migration, the original thesaurus database will be retired and maintenance of AGROVOC will continue on a production basis in the Workbench.

In the meantime, AGROVOC term codes and "termspells" have been widely used in agricultural portals and repositories worldwide. At FAO itself, AGROVOC terms have been used in AGRIS; in an International Portal on Food Safety, Animal and Plant Health; in an Emergency Prevention System for Transboundary Animal and Plant Pests and Diseases; in Geonetwork, a repository of geospatial information; and in the Electronic Information Management System, a workflow database used at FAO to track publications.

Although AGROVOC has not yet been used in its "ontological" form for production databases, it has been extensively used for research, most notably in the $\mathrm{NeOn}$ Project $_{13}$, an EU-funded project of 14.7 million Euros involving fourteen partners in seven countries for four years starting in March 2006. The NeOn Project aimed at providing "lifecycle support for networked ontologies" in large-scale, distributed applications.

FAO's role in the NeOn Project - carried out by the AIMS team in cooperation with FAO's fisheries department — was to implement a prototype Fish Stock Depletion Alert System in support of the long-term goal of sustainable fisheries. The task of the AIMS team was to integrate a diversity of data sources into a decision support system - sources ranging from land and fishing areas (identified using geographical coordinates), to biological entities (including family and species), fisheries commodities (using global statistical codes), fishing vessels (types and sizes), fishing gear (using a global classification scheme), and images from a variety of Websites. Related concepts needed to be aligned; water areas needed to be related to neighboring land areas. The objective was to federate the independent ontologies under a common queryable data infrastructure.

In 2003, a previous project in-house at FAO had attempted to build a comprehensive monolithic fishery ontology as a central focus for mappings from stand-alone databases, but work had bogged down with modeling issues, and the resulting con-

\footnotetext{
$12 \mathrm{http} / /$ protege.stanford.edu/

${ }^{13} \mathrm{http} / /$ aims.fao.org/website/ $\mathrm{NeON} / \mathrm{sub} 2$
} 
struct was impractical and unwieldly. The NeOn approach, in contrast, was that of a "network of ontologies." It assumed that datasets would continue to evolve within specialized communities of practice, each of which in turn reflected the diverse perspectives of managers, biologists, IT systems administrators, and thesaurus maintainers.

\section{User experience of AGROVOC and AIMS ontologies}

The AGROVOC Thesaurus was a loose, sprawling collection of terms added over of the course of many years by innumerable unnamed contributors and encompassing common and scientific names for bacteria, viruses, fungi, plants, and animals, as well as geographic names, acronyms, and chemicals. The terms all have something to do with agriculture or nutrition in a broad sense, but the thesaurus does not reflect any particular context, viewpoint, or application requirements. "Petroleum," for example, is narrower than "mineral resource" and related to "fuels"; the related term "oil spills" is narrower than "pollution," and "pollution" is narrower than "natural phenomena."

One important achievement of the re-engineering process of the past few years has been to "clean" the ontology by consolidating hundreds of top terms, linking hundreds of "orphaned" concepts, and correcting thousands of other inconsistencies.

The process of refining semantic relations, described above, has added more precise relationships, though the process has not been guided by an overarching standpoint - e.g., viewing the entities consistently from the standpoint of business, science, farming, or the environment. The semantic multivalence of the terms is augmented further by the subtle differences of perspective and interpretation introduced by their translation into nineteen languages.

Advanced reasoning, however, presupposes a commitment to an ontologically well-defined point of view. One user finds the effort to refine relationships useful in principle but hard to exploit in practice:

For our resource-discovery purposes, we cannot really apply the more refined relationships. I do not see how they can work - at least we do not have the technology to use them for resource discovery. You need an inference engine that can use them. Without an inference engine and a purpose, it is not clear what to do with them.

Another believes the effort is useful but explains that their particular application required relationships to be refined differently, so they ended up extracting a sub-set of AGROVOC concepts as a starting point and refining it into an ontology in their own particular way.

A recurring theme in user feedback is the case in which developers set out to create expert systems using well-engineered ontologies for text mining or decision support systems and ended up falling back on less sophisticated uses for the ontology such as simple query expansion and structured browsing. One FAO partner recounts the challenge of building a sophisticated ontology application with domain experts in the field: 
A group of extension officers in plant protection first tried to make a sophisticated portal on pesticides - a resource that extension officers could consult to help farmers diagnose plant diseases. They tried some complex solution and at some point, they completely gave up. They know the reality, they know their plants and all the relationships - the reality they know is so complex — but they couldn't use it to build an information system. They lacked the knowledge for creating a search assistant with an inference engine. The lesson we learned was that getting the various experts together, identifying the relevant material, and submitting it to the system, was actually more important than the highly codified system that resulted. In the end, we're talking here about references to just 1,000 research reports — and that is quite a lot for a specialized field! Once we identified those 1,000 reports, we did not need overly refined discovery methods.

One FAO technical officer with experience in ontology projects feels the requirements for reasoning functionality were never properly clarified:

The few ontologies in FAO are not exploited fully in terms of reasoning capability, and there are no real specific requirements for reasoning. The real requirements, like language independence and collaborative maintenance, do not require rules and reasoning. Maybe we should investigate whether we really want to have a basis for full-fledged ontologies. Maybe researchers were pushing for more functionality than really required.

Other users confirm that their needs are quite simple - better navigation, search refinement, or ranking hits:

We have used ontologies in vertical portals to index or classify things. We use OWL formats, but more like thesauri. With mappings, we can continue using legacy thesauri. We find we get better navigation; they help in ranking hits and refining searches.

One colleague in a FAO technical department would like to use AGROVOC to tag reports and publications:

Increasingly we have stuff to tag: meeting reports, publications, duty travels, case studies.

Much mundane, day-to-day stuff. If we had it "in AGROVOC," we could do interesting things. "Where are meetings duty travel reports, institutions, and Web pages we have done about, say, fungus?"

Fishery experts in the NeOn Project express enthusiasm about the potential of ontologies to guide decision-making but recognize that the methods may take a few years to mature. For the AIMS team, the project confirms that the maintenance of alignments within a "network of ontologies" is time-consuming and error-prone, especially between ontologies based on different underlying models (e.g., classversus instance-based) and between ontologies that are themselves independently evolving. Recognized bottlenecks are the lack of tools for automating such tasks and the lack of reliable corpi with which to test automatic alignment methods.

\section{AGROVOC as a "quarry" of terms}

The goal articulated for the Agricultural Ontology Server in 2001 was that of providing "building blocks" for application-specific ontologies. Feedback from users strongly confirms that this is indeed how AGROVOC is being used, only not for the 
sophisticated applications originally envisioned. In practice, AGROVOC serves as a quarry of conceptual blocks to extract as a starting point for customized vocabularies:

We need specific vocabularies in many areas. Making derivative products from AGROVOC — terms relevant for a particular area — is what people want to have: go one level down, slice up the pie with very specific terms in a particular area.

Sets of AGROVOC terms often provide a starting point for creating specialized portals about topics like "crop pests" or "bananas." The Organic Edunet 14 used AGROVOC as a starting point for their own set of categories, mapping to AGROVOC wherever possible and inventing the rest. It is simply more efficient to re-use an existing vocabulary than to try to invent one from scratch:

We need something between Yahoo and Dewey and more specific. It would take alot of discussion to come up with our own. We use taxonomies both for indexing and for creating the structure of Web pages. For each entry in the browsing structure, we want to have a query to the database using subject headings.

In its entirety, however, AGROVOC is simply too big:

Using all of AGROVOC is cumbersome - putting whole thing into peoples' hands is too much. We want to make a sub-vocabulary. We are moving towards full-text indexing and need vocabularies for very specific portals.

Given the wide range of audiences for which AGROVOC is used, however, the semantic multivalence of its terms is actually desirable. The Agropedia Project in India needs to customize browsing structures for users ranging from scientists to agricultural extension works and semi-literate farmers. Another user reports:

We have customers who produce portals for regional development - specific birds, sheep, things in meadows, how to manage meadows in specific ways. We need taxonomies to create a browsing structure for our portals, and not just from a scholarly perspective.

Many users see an inherent tension between centralizing quality control over AGROVOC maintenance with experts in the AIMS team as opposed to decentralizing control over the expansion of AGROVOC to user groups and language communities with their own local requirements:

I see a need for lots of country-specific AGROVOCs - for India, Brazil, etc. Everyone has very specific terminology. It is not doable to capture all of these variants in the central AGROVOC ontology. We need distributed vocabularies.

Decentralizing maintenance control, however, implies capacity development — instruction about ontological principles and training in the use of specific tools and procedures:

AGROVOC is understaffed for the task of maintaining AGROVOC, allowing new concepts without duplicating or creating a mess. One always has to check and think before entering a term - it is not a mechanical job for a clerk but involves brainware. KCEW could explain tagging as a capacity-building effort. This could be useful but would conflict with the maintenance task. There is possibly a built-in friction between the two roles.

$14 \mathrm{http} / /$ www.organic-edunet.eu/ 
Users see this as a crucial role for the AIMS team:

FAO provides AGROVOC to download and use, but just as important have been the people who provide support. This is extremely helpful! They bring new ideas. As a UN organization, FAO should have this role - to help solve problems.

Users also feel that decentralizing maintenance would free the vocabulary to grow more quickly:

AGROVOC is very strong, especially in geographic areas — we like it — but it evolves too slowly to keep pace with emerging research terms. Maybe we need vocabularies in a wiki or blog thing, like Wikipedia, where people can quickly post these things and start to adopt terms quickly — where terms can be proposed and used immediately.

That more sophisticated ontology applications imagined in the early 2000 s have not materialized in the AIMS user community has been, to some extent, both a barrier to understanding and a source of tension between visionaries and practitioners. Ontologies have been seen as bleeding-edge research — a noble undertaking but impractically complicated for the average implementer. The simpler and straightforward goals of today's Linked Data movement, however, are seen by many users as a crucial way forward. In this regard, the developments in the AIMS community have simply followed the trajectory of the wider Web world. It would seem that the goal of honing the precision of well-engineered ontologies stands at cross purposes with the goal of accommodating a broad diversity of language communities and user perspectives.

\section{Correcting the model for less precision}

Since the 2006 finalization of a metamodel for expressing a term-based thesaurus (i.e., AGROVOC) as an ontology of Concepts linked to Lexicalizations, the World Wide Web Consortium has finalized a W3C Recommendation for precisely this purpose: Simple Knowledge Organization System (SKOS) [8]. Indeed, a computer scientist from the AIMS team participated in the W3C Semantic Web Deployment Working Group which developed SKOS, and AGROVOC provided a key use case for the requirement that Labels (Lexicalizations) be defined as first-class resources [6]. It is indeed fortunate that AIMS team has not yet finalized the conversion of AGROVOC from thesaurus to ontology or promoted the URIs of its concepts, modeled as OWL classes, for use in Linked Data, because the shift to a SKOS metamodel can still be undertaken without breaking existing applications.

Figure 2 shows how AGROVOC can currently be expressed in SKOS: AGROVOC Lexicalizations (Terms) are modeled as instances of the class SKOS Label, AGROVOC Concepts as instances of the class SKOS Concept, and the AGROVOC Concept Scheme itself as an instance of the class SKOS Concept Scheme (see Fig. 2). This shift solves several problems with the 2006 AGROVOC metamodel, most crucially because SKOS provides a vocabulary for expressing the legacy thesaurus relationships between concepts not as ontologically strong sub-class relationships, but as ontologically weaker "broader" and "narrower" relationships. This is 


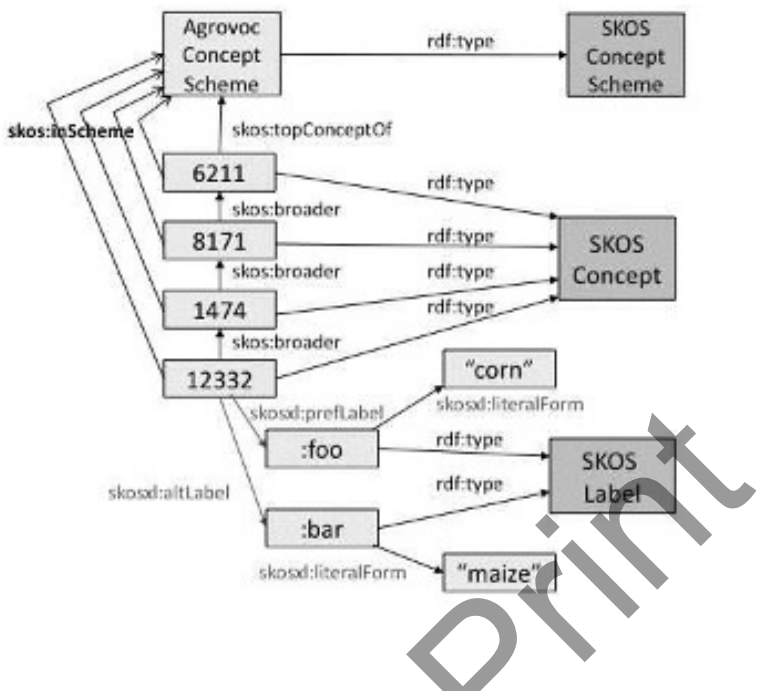

Fig. 2 AGROVOC modeled as a SKOS Concept Scheme (proposed)

more appropriate for AGROVOC because the mechanical translation of thesaurus terms into OWL classes violates the design principle of minimal ontological commitment. As explained by Thomas Gruber [3]:

An ontology should require the minimal ontological commitment sufficient to support the intended knowledge sharing activities. An ontology should make as few claims as possible about the world being modeled, allowing the parties committed to the ontology freedom to specialize and instantiate the ontology as needed. Since ontological commitment is based on consistent use of vocabulary, ontological commitment can be minimized by specifying the weakest theory (allowing the most models) and defining only those terms that are essential to the communication of knowledge consistent with that theory.

SKOS concepts make a minimal ontological commitment to the nature of concepts and of relationships between concepts. Constructs consisting of SKOS concepts do not support reasoning as extensively as do sets of tightly defined and constrained OWL classes, but they more faithfully reflect the flexible way that people actually think. SKOS concepts, by default lightly specified, prevent modelers from introducing false precision into their models, and they prevent inferencers from drawing unwarranted conclusions.

We have seen above that in practice, concepts are often extracted from AGROVOC, like building blocks from a quarry, for uses that more often than not are quite 
basic. Erring on the side of under-specifying concepts avoids imposing inappropriate ontological commitments and reduces the risk of their being reused incorrectly. Users of SKOS concepts in applications downstream do not inherit the transitivity and entailments of OWL sub-classing.

Declaring AGROVOC concepts as SKOS Concepts, on the other hand, does not preclude the use of OWL properties for defining relationships between concepts with more precision than the basic set of SKOS properties, e.g., as transitive, inverse, or symmetric. When appropriate, SKOS concepts may also be upgraded to OWL classes, with additional constraints, for use in local ontologies. (It is worth noting that the likewise lightly specified Dublin Core Metadata Terms are often upgraded locally from RDF into OWL properties, then more tightly constrained to support reasoning. As there are endlessly different ways to do this, the minimal commitment of the Dublin Core specifications in this regard is considered a basis of their success.) Defining AGROVOC in SKOS does not, in other words, preclude the development of applications that use reasoning.

Putting the Workbench onto a SKOS basis means that its deyelopers will be able to benefit from software libraries and interfaces being developed for what is already the most widely deployed standard for Linked Data vocabularies. This will, in turn, make the Workbench more attractive for the open-source development community. Users will be able to process the RDF representation of AGROVOC, or an extract thereof, not just with the Workbench but with any SKOS-enabled software. Use of the Workbench will not depend on support for a metamodel unique to AGROVOC.

The conversion into SKOS will also resolve another issue that has emerged as a problem for AGROVOC - the presence of "concepts" that should arguably be conceptualized as "instances." Examples include living species, chemicals, languages, and geographic place names, such as AGROVOC Concept 3253 ("Ghana"). In SKOS, every Concept is by definition an instance of the class SKOS Concept - in other words, every concept is by definition an instance, and the only question is whether there is a meaningful difference between "concept-like" instances and other, "non-concept-like" instances. Although it has been suggested that SKOS Concepts be reserved for "concepts" instead of "real-world" things — or for "universals" rather than "particulars" - such distinctions are not understood widely enough to provide a basis for consistent distinctions. By design, therefore, nothing in the SKOS data model prevents AGROVOC Concept 3253 ("Ghana") from being considered a SKOS Concept.

Forcing a distinction between classes and instances may, in fact, force ontological overcommitment. In order to map AGROVOC to an ontology for Aquatic Sciences and Fisheries Abstracts (AFSA), for example, the NeOn Project had to make AFSA comparable to AGROVOC by mechanically converting it into an ontology of OWL classes. On the other hand, while it seemed logical to the NeOn team that a species of fish be considered a class, and that actual fish be considered instances of that class, they found that when mapping to statistical time series, they needed needed to map species as instances. Indeed, the project team concluded "that the domain of interpretation of fisheries can contain entities as well as types of entities, and distinguishing them in a logically sound way would require a huge amount of 
fishery experts time, and only after they are organized in a team sided by ontology designers and are taught design tools adequately." [2] Thanks to their ontologically light specification, in other words, SKOS vocabularies can more safely and easily be mapped.

This ontologically more flexible approach to concept schemes also addresses a difficulty that has emerged in AIMS capacity-developing activities. AIMS team members holding seminars at FAO partner institutions report that words like "ontology" and "concept server" are perceived as "confusing," even "scary," and that the finer points of ontologies, such as the distinction between classes and instances, are lost on many audiences. The distinctions are, of course, hard to teach in part because they are hard to nail down or justify in reality. SKOS should be easier to teach, and with the rapid uptake of SKOS, AIMS trainers should benefit from the growing availability of tutorial materials.

The effort to refine AGROVOC concept relationships has underlined a need to standardize some frequently used properties such as "hasAcronym." The popularity of lightly defined concepts suggests, however, that the push to refine AGROVOC as a whole be given lower priority, moving forward, than the gradual extension of the concept set into new languages and subject areas. Mark yan Assemreports that the reluctance of vocabulary maintainers to complexify their vocabularies ontologically may be based on healthy "investment versus gain considerations," as it is not always clear how refinements improve performance and user support. He suggests that vocabulary developers follow the adage "no innovations without clear applications." 15

The AIMS namespace for AGROVOC currently defines 198 refined relationships, two-thirds of which constitute a "long tail" of properties used less than twenty times, or even just once or twice, as with "isAfflictedBy" or "hasBreedingMethod." The AIMS team will publish these properties as Linked Data, enabling their re-use in other projects, but the AIMS team will not have the resources to pursue their standardization in the global arena. Ideally, this task should be undertaken in the context of a standards organization, perhaps with the goal of starting with a manageable core of, say, fifteen popular and well-understood properties - a "Dublin Core" of thesaurus refinements. In the meantime, specifying all of the existing refinements as sub-properties of the original thesaurus relationships (Broader, Narrower, and Related) would allow an application to "dumb down" the refined relationships for simple purposes such as query expansion.

Guus Schreiber points out that vocabularies cannot simply be "merged" because they reflect a diversity of perspectives. Rather, the best one can realistically hope for is to make the vocabularies usable jointly by defining a limited set of mappings in a process of "vocabulary alignment." Published as Linked Data as a part of AGROVOC (or as a separate module), mapping assertions effectively increase the reach of AGROVOC concepts, allowing queries to be expanded to resources indexed with terms from related agricultural vocabularies such as the CAB Thesaurus (see above) or more general vocabularies such as Wordnet or the Library of Congress Subject

\footnotetext{
15 Personal communication.
} 
Headings. Facilitating the creation of such alignments has been identified as a new priority for the Workbench project.

\section{Networking, capacity development, and outreach}

A significant part of the AIMS initiative falls under the heading "capacity development" - building partnership among international colleagues through distributed teamwork, workshops, and training seminars in member countries or at headquarters. Capacity-developing efforts typically focus on the formation of information managers, local champions, and educators at regional universities and research centers ("training the trainers"), often with an effort to involve agricultural extension workers or reach out to farmers directly. Capacity development may involve on-site training sessions by FAO staff or research sojourns by visitors in Rome.

The AIMS team has helped build or provided training for regional initiatives such as the following:

-Red Peruana de Intercambio de Informaci on Agraria, a network of public and private institutions for supporting agricultural science and innovation in Peru with an emphasis on technical exchange and information management standards.

-The Kenya Agricultural Information Network, a three-year project funded by the UK Department for International Development, which among other things provided training in the use of metadata to participate in AGRIS.

- The Thai National AGRIS Center, established in 1980 as part of the Kasetsart University Central Library, which was an early adopter of the AGRIS application profile as the basis for merging content from twenty national research institutes and making it freely ayailable on the Web.

-The National Agricultural Research Information Management System (NARIMS) in Egypt, a bilingual Arabic-English Web portal for information about research in Egypt related to agriculture, which was developed in cooperation with FAO staff and using FAO tools and standards, notably an Arabic version of the AGRIS application profile. Starting in 2010, NARIMS data will be harvested by Near East Agricultural Knowledge and Information Network, a platform for agricultural research organizations in the wider Near East region and, from there, ingested into the central AGRIS database.

-The Global Forest Information Service ${ }_{16}$, a portal for information sources related to forestry, from maps and datasets to grey literature and journal articles.

The story of several related projects in India exemplifies the role that the AIMS team can play in developing capacity on several levels. Starting in 2002, the Indian Institute of Technology in Kanpur experimented with using the Web to help semiliterate farmers bypass intermediaries to sell their commodities online. The initial

${ }^{16} \mathrm{http}: / /$ www.gfis.net 
idea of promoting digital commerce failed for lack of uptake, but the project did confirm a need to transfer knowledge about crops (such as dal and sugar), farming methods (sericulture and pest control), and agrarian legislation from India's 11,000 or so $\mathrm{PhD}$-level agronomists to its 100 million farmers to address issues such as crop rationalization, declining soil fertility, the after-effects of chemical use, and pest pathologies.

The initiative enlisted the collaboration of village-level agricultural extension workers in bridging this gap and aimed at disseminating information in broadly consumable forms such as radio broadcasts, comic books, and SMS alerts, written or spoken in the rural vernacular. One strategy for making research outputs accessible to a broader range of participants was to tag available materials with familiar concepts, so parts of the AGROVOC Thesaurus were translated into Hindi and Telugu.

A larger National Agriculture Innovation Project, "Agropedia," 17 was launched in January 2009 to empower farmers and extension workers with crop- and regionspecific information and "accelerate technology-led, pro-poor growth and diffusion of new technologies for improving agricultural yield and rural livelihood." A brainstorming workshop with seventy participants of diverse background generated knowledge models reflecting scientific, clinical, and practical perspectives on the management of key crops such as rice, pigeon peas, and sorghum.

Taking AGROVOC concepts as a starting point, the participants used simple open-source software to define entities and relationships. Experienced ontologists from FAO helped apply standard naming conventions and map the emerging relationships to existing properties in AGROVOC. The workshop served both as a capacity- and a community-building experience. The resulting knowledge models link local terminology to standardized, language-independent concepts usable for tagging research outputs and learning materials, whether by manual metadata creation or automated keyword extraction, and to access those materials from a variety of perspectives.

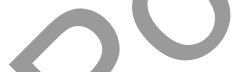

Fishing in a Sea of Agrovoc?

In 2004, an autoevaluation with focus groups at FAO identified the need for "a prolonged effort to monitor the departmental sites, put a coherent layer of metadata over the different information systems (building on already existing metadata), and do some quality assurance in order to bring some order to the FAO site and better index it." The evaluator reported that previous efforts to put order to the proliferating departmental sites "was never a pretty process; a lot of tension was involved between divergent departments. Everybody is so busy with service/divisional work that coordination is viewed as a burden."

There have been a few cases of successful cooperation between the AIMS team and technical departments within FAO, notably with Fisheries (in the NeOn Project)

${ }^{17} \mathrm{http} / /$ agropedia.iitk.ac.in 
and Forestry, involving primarily the use of AGROVOC for indexing, Agrifeeds for disseminating information about events, and the use metadata for describing departmental outputs. Overall, however, the observations made in 2004 appear still to apply five years later.

One technical colleague at FAO, however, offers a compelling metaphor for what might possibly be achieved in such a diverse institution:

\footnotetext{
There is absolutely a need for more communication between departments at FAO. Everything we do can be seen from multiple angles: Capacity Building, Research, Women and Development, Democracy. If we were swimming in a Sea of AGROVOC, and we were to cast our hook for Climate Change, what things might we pull up?
}

The same colleague argues that such an approach is essential for preserving and transmitting institutional knowledge in a faster and more mobile age:

There is quicker turnover now. With quicker staff turnover, institutional memory becomes a bigger problem. I used to be the youngest person in my department, but in the past three or four years, there have been more retirements. Who can tell me what meetings were held?

How might such a vision be achieved in practice? One well-developed model is offered by the VIVO service, managed since 2003 by the Cornell University Library as a structured view of information about people and academic resources at Cornell University.18 The sample of VIVO suggests the following lessons:

-Start small, with a few common content types - people, departments, courses, publications - and extend the supported types organically, based on growing relationships to people, activities, and organizations.

-Work with departments and administrators to promote a more uniform approach to self-reporting and demonstrating Return On Investment in the form of improved data consistency and higher public visibility.

-Invest data from departments and databases with as little manual intervention as possible, adapting automated ingest procedures to specific local data structures and using simple inferencing to enrich data records with information not explicitly encoded in the source databases (e.g., "member of life science field") and, where possible, enriching or replacing text values with URIs.

- Convert data into an open and consistent format, using explicit semantic relationships, and publish the data according to accepted Linked Data principles, avoiding a requirement that any one tool be globally accepted and anticipating instead the future availability of innovative alternatives.

-Present users with a clean, Google-like search box in recognition of the fact that people typically submit queries of just one or two words.

-Take the user from a single-word query to a page that assembles links clustered by type - people, events, publications, institutions, and topics - efficiently exposing the searcher to response sets of high quality and providing a structured browsing experience based on semantic relationships.

$18 \mathrm{http}: /$ vivo.cornell.edu 


\section{The global "coherence" of information about food}

The AIMS initiative sees itself as part of a broader movement for improving the management of, and access to, agricultural information. FAO is part of an initiative that has coalesced under the banner of Coherence in Information for Agricultural Research for Development (CIARD), the result of expert consultations held in 2005 and 2007.

CIARD presents a broader context in which AIMS can be effective. Where AIMS focuses on information standards, especially the AGROVOC thesaurus and AgMESbased application profiles, with AGRIS as a key implementer, CIARD represents a broader community, institutional base, and scope of action, with Task Forces on Advocacy, Capacity Building, and Content Management. The CIARD Content Management Task Force advocates the use of common standards for enabling the integration of information across institutions. The CIARD Pathways to Research Uptake offer concrete advice on broader issues, such as licensing and open access, techniques for retrospective digitization, policies for sustainable repositories, digital preservation, the exchange of information about news and events, and effective Website management (Web 2.0, search engine optimization, social media, and the use of Web analytics).19

The notion of "coherence" fits beautifully with the message of Linked Data. We live in a diverse and rapidly evolving world in which it is unrealistic to expect that interoperability can be tightly coordinated on the basis of mandatory data formats and specific technical solutions, whether by "lock-step" agreement among big institutions or by the de-facto dominance of specific software platforms. RDF provides an open-ended data model that explicitly avoids requiring that providers information in identical formats - a goal which can only remain, in the best of circumstances, elusive.

Rather, the watchwords of this more loosely-coupled vision of interoperability are "alignment," "harmonization," and "partial understanding." The best we can hope for is "coherence" in the underlying data itself — to ensure that the data can be expressed as, or translated into, RDF triples that can be coherently merged on the basis of shared descriptive properties, shared value vocabularies, and shared resource identifiers. The language-neutral nature of URIs turns vocabularies such as AGROVOC into platforms for extending concept schemes into new language areas.

History shows that all technology is transitional. Most of the applications and data formats we use today will become obsolete in the coming decade. RDF triples represent knowledge in the form of a simple sentence grammar, using noun-like classes and verb-like properties to make statements about things in the world statements that are expressible in, and freely convertible among, multiple concrete syntaxes.

As of 2010, there are no other compatable models for representing knowledge with the uptake and traction of RDF. For the foreseeable future, RDF offers our best hope for "future-proofing" our cultural and scientific memory. As our applications

${ }^{19} \mathrm{http} / /$ www.ciard.net/index.php?id=607 
and formats inevitably lapse into obsolescence, we can only hope to retain the ability to interpret what remains. We must ensure that information about so existentially vital topics as food and nutrition be expressed in a form that we can flexibily re-use today and pass to the next generation tomorrow.

\section{References}

1. Brickley, Dan. 2003. Missing isn't broken: data validation and freedom on the Semantic Web. FOAF Project Blog, http://blog.foaf-project.org/2003/07/missing-isnt-broken-datavalidation-and-freedom-on-the-semantic-web/.

2. Caracciolo, Caterina. 2009. D7.2.3. Initial Network of Fisheries Ontologies. NeOn Project. http://www.neon-project.org/web-content/images/Publications/neon 2009 d723.pdf

3. Gruber, Thomas. 1995. Toward Principles for the Design of Ontologies Used for Knowledge Sharing. International Journal Human-Computer Studies 43(5-6]: 907-928.

4. Hepp, Martin. 2007. Possible Ontologies: How reality constrains the development of relevant ontologies, Martin Hepp. IEEE Internet Computing 11(1): 90-96.

5. Independent External Evaluation of FAO. 2007. Rome: FAO. $\mathrm{ftp} / / \mathrm{ftp}$.fao.org/docrep/fao/meeting/012/k0827e02.pdf.

6. Isaac, Antoine, Jon Phipps, Daniel Rubin. 2009. SKOS Use Cases and Requirements. [W3C Working Group Note, 18 August 2009]. http://www.w3.org/TR/skos-ucr/\#UC-Aims.

7. McGuiness, Deborah, Frank van Harmelen, eds. 2004. OWL Web Ontology Language Overview. [W3C Recommendation 10 February 2004]. http:/www.w3.org/TR/owl-features/.

8. Miles, Alistair, Sean Bechhofer, eds. 2009. SKOS Simple Knowledge Organization System Reference. [W3C Recommendation, 18 August 2009]. http://www.w3.org/TR/skos-reference/.

9. Sauermann, Leo, Richard Cyganiak. 2008. Cool URIs for the Semantic Web [W3C Interest Group Note 03 December 2008]. http//www.w3.org/TR/cooluris/.

10. Soergel, Dagobert, Boris Lauser, Anita Liang, Frehiwot Fisseha, Johannes Keizer, and Stephen Katz. 2004. Reengineering thesauri for new applications: the AGROVOC example. Journal of Digital Information 4(4). http:/journals.tdl.org/jodi/article/view/112/111.

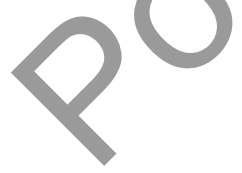

